\title{
Individual Differences in Sensitivity to Nicotine: Implications for Genetic Research on Nicotine Dependence
}

\author{
Ovide F. Pomerleau ${ }^{1,2}$
}

\begin{abstract}
Recent evidence suggests that cigarette smoking has a heritability index around $53 \%$. While related research on underlying mechanisms also supports the idea that genetic factors contribute to nicotine dependence-as well as to cofactors such as substance use and mood disorders - the nature of the behavioral traits and biological capacity for reinforcement that constitutes vulnerability to nicotine dependence is not well understood. The present review explores the problem of why some people become highly nicotine dependent, others develop a pattern of occasional use, and still others avoid the drug entirely despite extensive exposure and widespread experimentation with tobacco in the population. Recent research - both infrahuman and human-suggests that vulnerability to nicotine dependence is related to high initial sensitivity to nicotine and that the development of tolerance is more rapid and self-administration more extensive in such individuals. Relevant findings from neuroscience and biobehavioral research are reviewed in order to identify variables and methodologies that might improve the reliability and validity of behavioral and molecular genetic studies on cigarette smoking. The integration of research in these areas may lead to new insights in the understanding of nicotine dependence as well as to improved techniques for prevention and treatment.
\end{abstract}

KEY WORDS: Drug sensitivity; dependence; tolerance; nicotine; smoking.

\section{INTRODUCTION}

Initiation into tobacco use was once so common as to constitute a growing-up ritual, at least among male adolescents. In many parts of the world, this is still the case. That social factors in initiation are relevant is indicated by the fact that the smoking status of friends is a good predictor of initiation of smoking (Bewley et al., 1974). Smoking by parents and by older siblings is also predictive, however, suggesting the possible contribution of genetic factors as well. More than 30 years ago, Fisher (1958a, b) explored this possibility and noted that concor-

\footnotetext{
${ }^{1}$ Behavioral Medicine Program, Department of Psychiatry, 475 Market Place, Suite L, University of Michigan, Ann Arbor, Michigan 48105. Fax: (313) 998-6443.

${ }^{2}$ Preparation of this manuscript was supported by Grant DA06529 from the National Institute on Drug Abuse.
}

dance for smoking behavior was higher in monozygotic (MZ) twins than in dizygotic (DZ) twins. Some of the twins had not been reared together, indicating that the similarity between the twins was not exclusively the result of environmental influence. These observations have been replicated by numerous investigators: Hughes, for example, identified 18 twin studies in which concordance rates for smokers were consistently greater for $M Z$ than for $\mathrm{DZ}$ twins, with a mean heritability estimate of $53 \%$ for tobacco use, ranging from 28 to $84 \%$ (Hughes, 1986, Table 1).

Other twin studies have demonstrated that genetic factors not only contribute to the initiation of the smoking habit (Eaves and Eysenck, 1980; Hannah et al., 1984) but also influence age of onset of smoking and number of cigarettes smoked per day 
(Heath and Martin, 1993) as well as persistence of smoking and intensity of smoking - with significant genetic variation evident for both light and heavy smoking though not for moderate smoking (Carmelli et al., 1992; Heath and Martin, 1993). Since alcohol use and caffeine consumption are also associated with smoking, Carmelli, Swan, and associates (Carmelli et al., 1990; Swan et al., 1990) recalculated heritability estimates for smoking, taking the correlation among these behaviors into account: Before adjustment, genetic contribution for smoking, alcohol, and coffee use accounted for 53, 36 , and $45 \%$ of the variance, respectively, whereas after adjustment, the corresponding estimates were 35,29 , and $36 \%$-smaller but still significant.

Recent research in molecular genetics suggests that there may also be commonalities at the level of gene products and, mechanisms of action for various addicting substances, among them nicotine and alcohol (Collins 1990). Though the findings are still equivocal, a number of studies have demonstrated increased prevalence of the $A 1$ and B1 alleles of the dopamine receptor gene (DRD2) in smokers (Comings et al., 1993; Noble, 1993), in alcoholics (Noble, 1993; Uhl et al., 1992), and in polysubstance users (Smith et al., 1992). Not only have commonalities in the use of tobacco, alcohol, and caffeine over the normal range of consumption been identified using twin studies (Swan et al., 1994), but also significant genetic associations between smoking and clinical depression (Kendler et $a l ., 1993 b)$ and alcohol use and clinical depression (Kendler et al., 1993a) have been demonstrated, adding further support to the molecular studies.

The above findings clearly indicate that genetic factors contribute to smoking (and to related substance use and mood disorders) in important ways. The nature of the behavioral traits and biological capacity for reinforcement that comprise vulnerability to nicotine dependence is not well understood. It is evident, however, that some people seem destined to become smokers because of strong biological reasons for smoking, irrespective of environmental factors: For instance, at the time of the first exposure, they may be less reactive to nicotine's toxic effects and/or they may be more responsive to nicotine's reinforcing effects. On the other hand, from the onset, never-smokers may be more sensitive to nicotine's noxious effects or they may develop tolerance to nicotine's aversive actions more slowly or they may simply fail to derive sufficient reinforcement from nicotine to sustain the habit. These possibilities have not been examined systematically, and the challenge for behavioral and molecular genetics will be to design studies that can take advantage of the findings of recent biobehavioral and neuroscience research.

The present paper explores the problem of why-despite extensive exposure and widespread experimentation with tobacco-some people become highly nicotine dependent, others develop a pattern of occasional use, and others avoid it entirely. A central premise is that, because initial reinforcement consequences set the stage for subsequent nicotine use, individual differences in sensitivity/reactivity to nicotine constitute a critical element in susceptibility. Following an introduction to basic terminology and a review of current models of the relationship between sensitivity and dependence, the report focuses on a selective examination of recent research on individual differences on sensitivity to nicotine-both infrahuman and human-in the process identifying pertinent variables and methodologies. Subsequently, the implications for genetic research will be taken into account, by way of encouraging a synthesis that might engender new insights in the understanding of smoking.

\section{TERMINOLOGY AND BASIC CONCEPTS}

Terminology in the 1988 Surgeon General's Report, The Health Consequences of Smoking: Nicotine Addiction (USDHHS, 1988, pp. 44-45, 289), is employed. Sensitivity indicates a situation in which, at initial exposure, there is greater response to a specified drug dose or in which a smaller drug dose suffices to elicit a specified level of response. Tolerance is defined in two ways: (a) as decreased reactivity to a given dose of the drug as a result of repeated administration over time and (b) as the need for a larger dose to maintain the effect originally obtained. Tolerance is further differentiated into four subtypes: Dispositional (pharmacokinetic) tolerance refers to accelerated drug elimination (i.e., increased distribution and/or clearance) as a mechanism for explaining diminished effects after repeated dosing. Behavioral tolerance describes the decreased effect of a given dose of a drug as a result of compensatory behaviors that reduce adverse effects on performance or activity. Conditioned tolerance refers to responses elicited by 
association with drug administration-typically drug-opposite reactions that reduce drug effects. Functional (pharmacodynamic) tolerance characterizes loss of responsivity to dosing observed when dispositional and behavioral tolerance can be ruled out; more specifically, it refers to a condition in which drug concentration at the receptor siteapproximated in humans by plasma concentration of the drug - produces a smaller effect as a result of exposure. If functional tolerance develops within one or two exposures (i.e., during the period of drug agonist activity), it is referred to as tachyphylaxis or acute tolerance. Tolerance that persists after prolonged exposure is called acquired, or chronic tolerance.

Drug dependence is characterized as a process associated with regular exposure to a psychoactive substance in which neuroadaptations leading to a cycle of escalating tolerance and increased self-administration occur until some asymptote is reached (Alexander and Hadaway, 1982). If the cycle is interrupted, by either withholding the drug or blocking its action with an antagonist, the resulting disequilibrium is manifested by a withdrawal syndrome, with characteristic signs and symptoms; readministration of the drug provides relief unless its action is opposed by an antagonist.

Tobacco smoking fits this general description of addiction quite well: Nicotine is the major psychoactive agent in tobacco, and after initiation, smoking behavior increases over a number of years, eventually reaching a point at which plasma nicotine levels stabilize and are maintained or "regulated" within characteristic limits (Kozlowski, 1980; Russell, 1978). If plasma concentration falls below a certain level-different for each individual-smoking increases; if nicotine exceeds a certain level, toxic symptoms appear and smoking (nicotine self-administration) is reduced. Once entrained, these processes are highly resistant to change, for after a period of abstinence from smoking, there is a strong desire to smoke ("craving") along with the aversive symptoms that characterize withdrawal (Killen et al., 1988; Pomerleau et al., 1983). There is some disagreement about the exact percentage (Hughes et al., 1987), but estimates suggest that from 50 to $80 \%$ of smokers can be classified as nicotine dependent (American Psychiatric Association, 1994) by scoring positive on at least three of the following in the same 12-month period (Greden and Pomerleau, in press): (1) tolerance, manifested by decreased effect of a given dose and/ or increased dosing to produce the same effect; (2) withdrawal after a period of abstinence; (3) smoking a greater amount or over a more extended period than intended; (4) persistent desire to smoke and/or unsuccessful efforts to cut down; (5) considerable time spent obtaining or using tobacco; (6) important social, occupational, or recreational activities given up or curtailed because of smoking; and (7) continued smoking despite knowledge of risks to health.

\section{THE EXPOSURE MODEL OF TOLERANCE AND DEPENDENCE}

Only one-third to one-half of those who experiment with tobacco go on to smoke regularly (McNeil, 1991). A commonly accepted explanation for why the remainder escape addiction is that these people have high innate sensitivity to nicotine and, as a result, encounter aversive effects on initial use, thus limiting further experimentation (Silverstein $e t$ $a l ., 1982$ ); with no further exposure to nicotine, according to this conceptualization, there is no opportunity to develop tolerance. On the obverse side, people with less sensitivity to nicotine experience fewer unpleasant effects and are more likely to continue smoking (Friedman et al., 1985). Because inception of smoking is typically aversive (Bewley et al., 1974), initial use is presumed to be maintained by social reinforcement such as peer pressure (Friedman et al., 1985; Hirschman et al., 1984; Presti et al., 1992). After a certain critical exposure, however, dependence develops and the habit is now maintained by the avoidance of nicotine withdrawal (McNeil, 1991).

Furthermore, depending on environmental conditions, individuals become differentiated into heavy smokers or light smokers based on their exposure to nicotine; thus, heavy smokers are characterized as highly nicotine dependent because they typically have difficulty abstaining, are highly tolerant to nicotine, and exhibit greater withdrawal upon discontinuation of smoking than light smokers (Killen et al., 1988; Pomerleau et al., 1983). Since chronic tolerance is seen as a consequence of the extent of nicotine exposure (Gurling et al., 1985), this explanation of individual variability in smoking patterns has been called the "exposure model" (Pomerleau et al., 1993a). Whether an individual smokes or not is the result of initial sen- 
sitivity to nicotine, but degree of dependence is determined by exposure to nicotine under the influence of environmental variables such as social support. A diagram of the exposure model is provided in Fig. 1.

Because of its plausibility and also because of the difficulty of observing initiation of smoking directly, key elements of the exposure model have yet to be validated. For example, the critical amount of exposure to nicotine needed to differentiate tobacco users into light and heavy smokers and to establish degree of dependence cannot be specified. Further, though the exposure model holds that people who are more sensitive to nicotine's aversive effects are less likely to become smokers, the empirical evidence for this assertion is weak, and the relationship between initial sensitivity to nicotine and tolerance is not as obvious as the model would imply. The assumption that "nicotine exposure is the engine that drives tolerance" (Pomerleau et al., 1993a) has been questioned (see Shiffman, 1991b). The two sections that follow review recent neuroscience and biobehavioral research findings with respect to individual variability in the initiation of smoking and the development of tolerance and dependence.

\section{Molecular and Infrahuman Studies}

For over a decade, Collins and his associates have used inbred mouse strains to explore the relationship between individual differences in sensitivity to nicotine and the use of tobacco. From a genetic perspective, each individual mouse can be seen as the equivalent of a human monozygotic twin. The focus of recent studies has been to determine whether genetic factors regulate first-dose sensitivity to nicotine and the subsequent development of tolerance; a secondary objective has been to find out whether these effects were the result of changes in number or affinity of brain nicotine receptors (Collins and Marks, 1989). The research has taken advantage of the fact that nicotine affects a wide variety of behavioral and physiological processes that are readily measurable, including learning, locomotor activity, body temperature, respiration rate, heart rate, acoustic startle response, seizures, and adrenal hormone release.

In a recent analysis of nicotine sensitivity in 19 inbred strains of mice, Marks et al. (1989b) revealed extensive differences in initial sensitivity to nicotine on key parameters such as the $\mathrm{ED}_{50}$ (the nicotine dose that elicits $50 \%$ of maximum possible change in a given measure of activity). Two factors-body temperature/activity and sensitivity to nicotine-induced seizures-accounted for $69 \%$ of the variance, suggesting that a limited number of genes were operative in determining initial sensitivity to nicotine and that two specific gene pools might be responsible for the observed effects.

In a related experiment, Marks et al. (1989a) measured the binding number (maximal number of receptors, $B_{\text {max }}$ ) and binding affinity (degree of bonding between drug and receptor, expressed as a disassociation constant, $K_{\mathrm{D}}$ ) for receptors in eight brain regions for the 19 strains that had exhibited wide differences in initial sensitivity to nicotine. The mouse brain contains at least two nicotine receptor classes, one exhibiting a high affinity for $\left[{ }^{3} \mathrm{H}\right]$ nicotine and the other for $\alpha-\left[{ }^{125} \mathrm{I}\right]$ bungarotoxin [BTX] in radioligand binding assays (Marks and Collins, 1982). Binding affinity did not differ systematically (for either nicotine or BTX receptors) in the eight brain regions among the 19 strains, but the strains did vary in receptor number, with significant differences in receptor number in midbrain, hindbrain, hippocampus, hypothalamus, and colliculus across strains. Mice with a greater number of nicotine-receptor binding sites were more sensitive to nicotine in that they required lower doses of nicotine to elicit a particular response; this was indicated by an inverse relationship $(r=-.62)$ between initial sensitivity, using $\mathrm{Y}$-maze rears/ crosses and body temperature as indicators, and nicotine receptor number in the different strains; there was also an inverse relationship $(r=-.63)$ between seizures and BTX-receptor number. Differences in receptor number accounted for approximately $36 \%$ of the variance in sensitivity to nicotine.

The development of tolerance was studied in a similar manner. Because Collins and associates suspected that tolerance to nicotine might be linked to initial sensitivity, mouse strains known to differ widely in sensitivity to nicotine were selected and were infused chronic saline or nicotine over 10 days in order to engender tolerance (Collins and Marks, 1989). Some strains developed tolerance at the lowest infusion doses, but others developed tolerance only at the highest doses; the strains also differed in the maximal amount of tolerance evinced. The critical finding was that there was a 

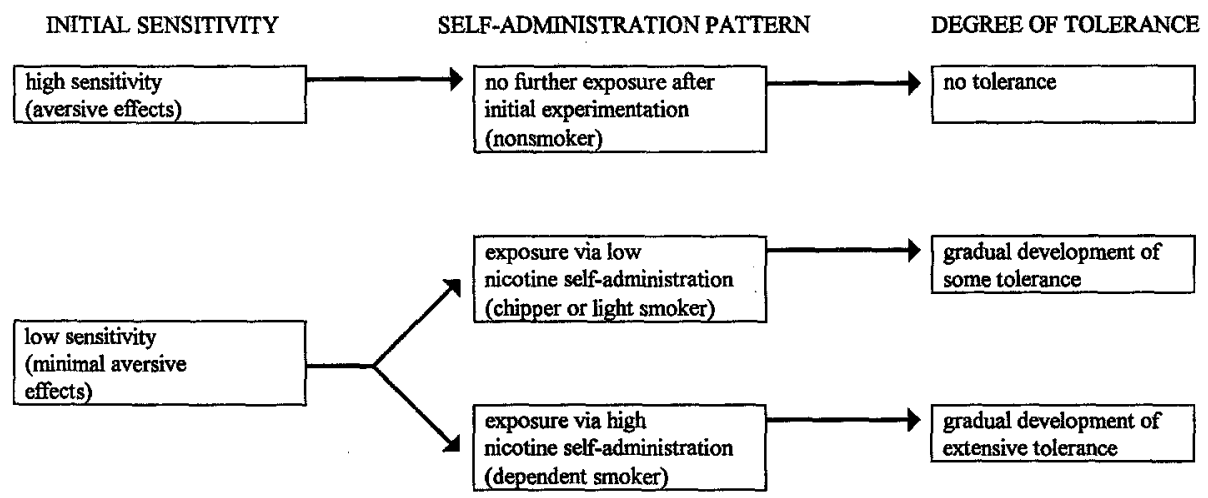

Fig. 1. Diagram of the exposure model of tolerance (from Pomerleau et al., 1993a).

strong positive correlation $(r=.86)$ between the initial sensitivity to nicotine and the minimal infusion dose required to elicit a shift in the doseresponse curve (i.e., the lower the dose required to elicit a given effect, the lower the dose required to induce a given degree of tolerance). The general conclusion was that the strains with greater initial sensitivity to nicotine were the ones that developed tolerance to nicotine more readily and to a greater extent.

Tolerance to chronic administration of nicotine was dose dependent and corresponded to an increase in nicotinic and BTX binding (Marks et al., 1986). In light of the observation that greater receptor number indicated greater sensitivity to nicotine in different mouse lines, the up-regulation of the nicotine receptor (Ochoa et al., 1990) was puzzling. Collins and Marks (1991) attempted to explain the association between the development of tolerance (decreased reactivity) and an increased receptor number as the result of receptor desensitization brought about by chronic dosing. It is known that nicotine functions as a mixed agonistantagonist, in that nicotinic receptors are desensitized as part of the process of agonist binding following nicotine administration and that this effect persists much longer for nicotine than is the case for acetylcholine, the endogenous ligand for the nicotine receptor. Sustained inactivation of the receptor due to chronic nicotine infusion -the functional equivalent of receptor blockade-likely increases receptor number due to stimulation of neuronal synthesis of new receptors. Thus, according to Collins and Marks, the phenomenon of nicotine tolerance might be explained as a process by which receptor desensitization (i.e., reduction in the number of activatable receptors) outstrips compensatory receptor synthesis. Various alternative explanations are being explored as well, including the possibility that some of the induced nicotine receptors are dysfunctional. Moreover, the characteristics of the receptor-binding assay used in the research by Collins and Marks (1989), are being assessed: The assay detects a single, high-affinity nicotine binding site, and molecular genetic studies have revealed the existence of several nicotine receptor subtypes, differentially distributed in the rat brain (Goldman et al., 1987). Should one of these receptor subtypes be critical to the regulation of tolerance to nicotine, and should mouse strains differ in either number or regional distribution of these receptor subtypes, this might provide a parsimonious explanation of differential tolerance development.

Receptor changes may be critical for understanding the development of tolerance in people as well. For example, the recent observation that nicotine binding is greater in selected brain regions of smokers at autopsy, compared with nonsmokers (Benwell et al., 1988), suggests that similar processes are operative at the human level. The significance of the experiments with inbred mouse lines is that it provides a model for identifying the reactions that set the stage for subsequent nicotine intake and susceptibility to dependence in the neophyte smoker. The adequacy of the approach is suggested by preliminary data indicating that the strains that were most sensitive to nicotine initially-and that developed the most tolerance following chronic exposure--were the ones that con- 
sumed the most nicotine in a drinking solution (Collins and Marks, 1991), suggesting that the mice differed with respect to some factor related to the reinforcing effects of nicotine. Overall, these results indicate that initial sensitivity to nicotine may presage not only the development of tolerance but the extent of subsequent self-administration-a key element in dependence. Further confirmation will be required, but the trends emerging from the inbred mouse studies contradict the conventional wisdom that degree of tolerance and dependence are simply the result of differential exposure to nicotine; they suggest, instead, than preexposure differences in sensitivity play a much more critical role in determining dependence that previously suspected, a theme that is developed further in the next section.

\section{Human Studies}

Some key tenets of the conventional exposure model were summarized recently by Perkins et al. (1993, p. 375): "One view of the onset of regular smoking suggests that repeated exposure to nicotine gradually leads to reduced magnitude of its effects, including subjective effects. This reduction in drug effects with increasing drug exposure is termed chronic tolerance. The onset of chronic tolerance to nicotine may subsequently lead to greater smoking in an effort by the smoker to continue to obtain the same magnitude of reinforcing effects of nicotine." The adequacy of this model for explaining smoking is brought into question, however, by numerous "anomalies." For example, Russell (1989) observed, with respect to recruitment to smoking, that it is still unclear whether the disappearance of nausea and other aversive effects following a period of experimentation with smoking can be attributed to the development of chronic tolerance. He noted that, in studies using animals, chronic tolerance to nicotine develops after a few days of steady intravenous infusion. As Benowitz (1991) has shown, however, the pharmacokinetic profile of nicotine administration is quite different in smokers, consisting of a sharp rise in plasma nicotine concentration, with arterial levels peaking as high as $100 \mathrm{ng} / \mathrm{ml}$ (about three times the venous concentration) while the cigarette is being smoked, followed by a rapid decay (half-life, 2 to $3 \mathrm{~h}$ ); thus, smoking produces a peak/trough pattern of chronic nicotine exposure- a pattern associated with potent physiological and behavioral effects (Balfour, 1993). The only study Russell could find that directly compared reactivity in abstinent chronic smokers and nonsmokers found no evidence for chronic tolerance, in that there were no differences between the two groups in sensitivity to the effects of an intravenous nicotine probe with respect to heart rate, blood pressure, and electroencephalographic changes (Murphree, 1979). Furthermore, earlier investigations by West and Russell (1985; 1988) had also noted that more dependent smokers-smokers who experienced greater withdrawal during abstinence-were actually more sensitive to the first cigarette smoked after $24 \mathrm{~h}$ of abstinence. The significance of these observations is that they explicitly link tolerance and degree of nicotine dependence in smokers to individual differences in sensitivity to nicotine that may have predated exposure, rather than to degree of exposure after initiation into smoking.

The recent development of several noninvasive methods for administering controlled doses of nicotine in a manner that mimics the sharp rise and fall of plasma nicotine from cigarette smoking (Pomerleau et al., 1989) has provided the opportunity to explore the phenomenon of sensitivity to nicotine much more systematically than previously feasible at the human level. Not only can the dose and pharmacokinetics of the nicotine delivery system be manipulated more readily than for cigarette smoking, but the potentially confounding contributions of the several thousand compounds in tobacco smoke are eliminated. Further, the vehicle for nicotine administration is novel for both smokers and nonsmokers, thereby minimizing the contributions of habituation (including behavioral and conditioned tolerance) to the dosing vehicle. These new methods may be of great help in elucidating the genetically transmitted behavioral and physiological characteristics that contribute to susceptibility to nicotine dependence.

Pomerleau et al. (1993b) conducted an initial exploration of the effects of nicotine on heart rate and blood pressure in 10 smokers and 10 lifetime nonsmokers (no sustained use of tobacco at any time). The approach used a brief, intranasal dose of nicotine (administration time, $<10 \mathrm{~s}$ ) as a probe for studying individual differences in sensitivity to nicotine. Nicotine delivery was via an airbrush used as aerosolizer, with dosage based on precise, electromechanical control of intranasal spray duration 

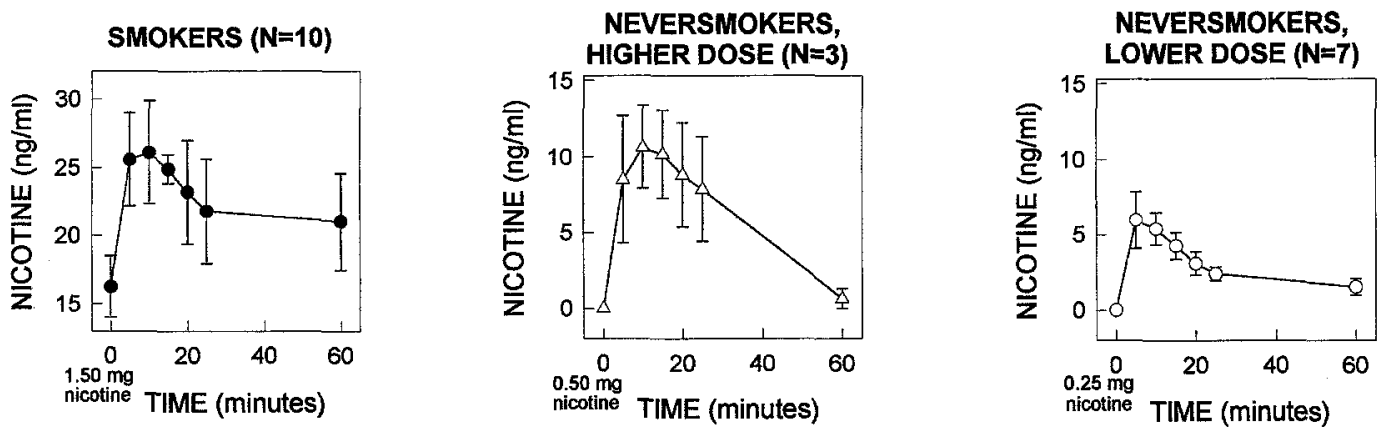

Fig. 2. Mean plasma nicotine \pm SE over time following administration of a fixed dose of nicotine via intranasal aerosol. Left, 10 smokers dosed with $1.50 \mathrm{mg}$ nicotine; middle, 3 never-smokers dosed with $0.50 \mathrm{mg}$ nicotine; right, 7 never-smokers dosed with $0.25 \mathrm{mg}$ nicotine (from Pomerleau et al., 1993b).

[see intranasal nicotine aerosol device (Pomerleau et al., 1992a)]. All smokers abstained from smoking for $1 \mathrm{~h}$ (constituting minimal deprivation) and received $1.5 \mathrm{mg}$ of nicotine. The first three neversmokers received $0.5 \mathrm{mg}$ of nicotine, and since all three experienced nausea, the remaining seven received $0.25 \mathrm{mg}$ of nicotine. As shown in Fig. 2, mean peak plasma nicotine increment for the 10 smokers was $11.5 \mathrm{ng} / \mathrm{ml}$. For the three never-smokers who received a $0.5 \mathrm{mg}$ dose, the peak increment was $12 \mathrm{ng} / \mathrm{ml}$; for the seven never-smokers who received a $.25 \mathrm{mg}$ dose, it was $7.5 \mathrm{ng} / \mathrm{ml}$. These results indicate that, at least for punctate dosing, nicotine accumulation in plasma per unit dose administered varied considerably between the groups: the ratio (mean plus or minus the standard error of the mean) of peak plasma nicotine level $(\mathrm{ng} / \mathrm{ml})$ to dose administered (mg) was $6.69 \pm 1.51$ in smokers and $28.08 \pm 3.88$ in never-smokers, a highly significant difference. Disparity in plasma nicotine accumulation between smokers and nonsmokers has also been observed by Srivastava et al. (1991), who noted that plasma nicotine concentrations in neversmokers were twice those of smokers following application of a $30-\mathrm{mg}$ transdermal nicotine patch. (Patches were presumed to have identical bioavailability.)

One interpretation of these findings is that smokers exhibited smaller plasma nicotine accumulation (i.e., evinced greater pharmacokinetic tolerance) than never-smokers due to greater volume of initial distribution; another possibility, though, is that smokers might have diminished bioavailability through some mechanism involving differential vasoconstriction or diminished nicotine trans- fer across the nasal mucosae. While the finding of greater accumulation of nicotine in plasma of never-smokers was certainly in keeping with the common observation that never-smokers experience aversive effects such as nausea and dizziness at lower doses of nicotine than smokers, a proper evaluation of pharmacodynamic (functional) tolerance-which reflects the effects of nicotine at brain nicotinic receptors-necessitated taking into account the differences in level of nicotine.

Figure 3 examines these differences by comparing plasma nicotine increment (peak concentration minus baseline concentration) with heart rate and blood pressure increments (peak response minus baseline response) for both smokers and neversmokers. [The peak for nicotine concentration was compared with the peak value for the response of interest in the analyses in order to eliminate the temporal disassociation between effects of nicotine at the receptor level - manifested by physiological responses proportionate to the arterial nicotine concentration - and the change in nicotine concentration in venous circulation, which lags several minutes behind fluctuations in arterial concentration (Porchet et al., 1987)]. Peak physiological reactivity for each subject was divided by the subject's peak nicotine increment (which, in venous blood, was reached 5 to $10 \mathrm{~min}$ later). Thus, differences in pharmacodynamic sensitivity between smokers and never-smokers were compared on the basis of their reactivity per unit plasma nicotine, thereby canceling out the contribution of pharmacokinetic differences between the two groups. Finally, in order to take into account customary nicotine intake and, by extension, nicotine depend- 


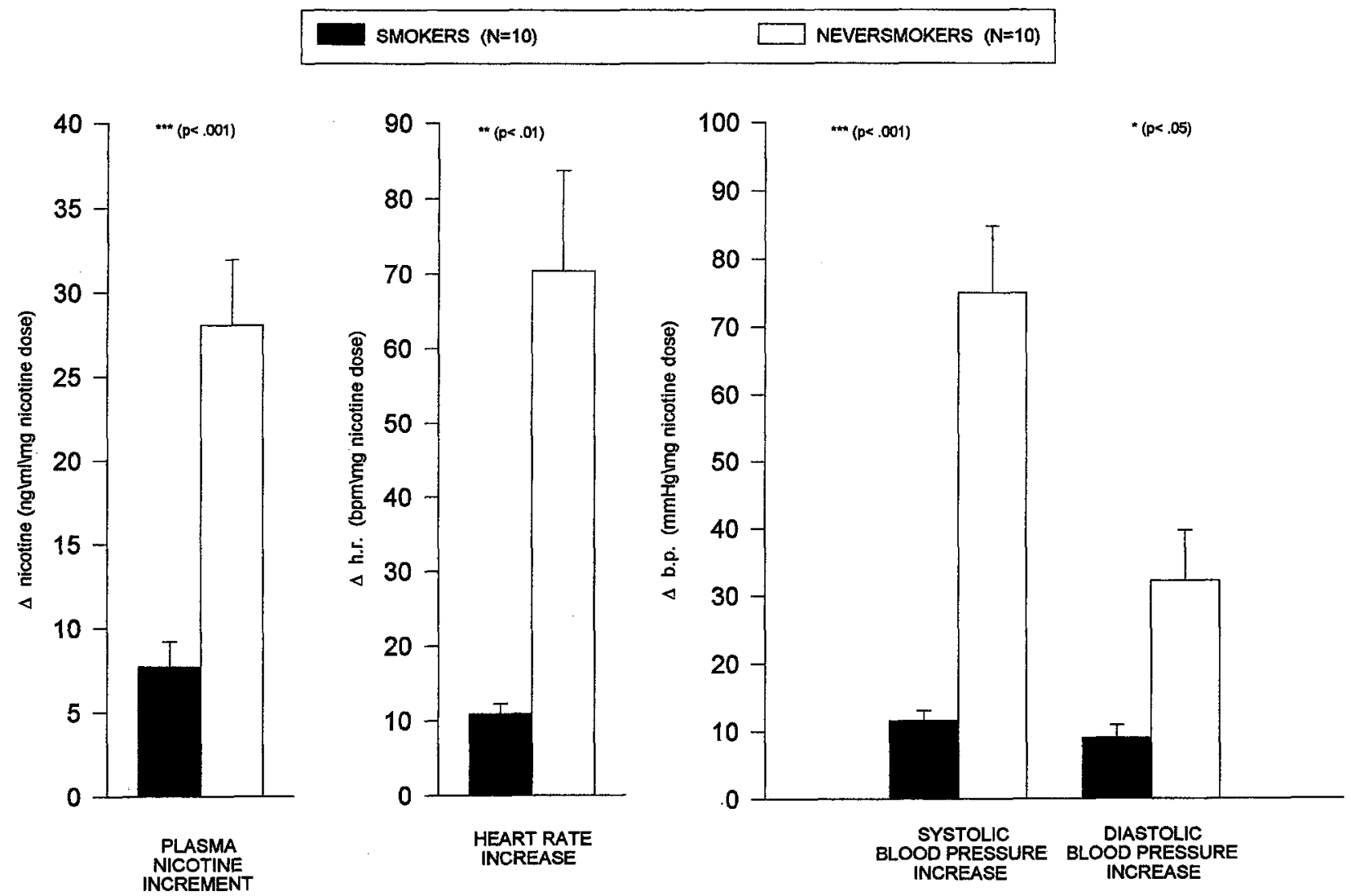

Fig. 3. Peak plasma nicotine increment and peak physiological response per unit nicotine dose administered (mean $\pm S E$ ).

ence (Pomerleau et al., 1983), smokers and neversmokers were classified by cotinine level [cotinine is a major metabolite of nicotine with a half-life of 18-20 h (Benowitz, 1991)]; values were obtained from samples obtained prior to nicotine dosing. As shown in Fig. 4, smokers varied considerably from one another, with cotinines ranging from less than 50 to nearly $400 \mathrm{ng} / \mathrm{ml}$; never-smokers, on the other hand, grouped in a column directly above the $0 \mathrm{mg} / \mathrm{ml}$ level. The ratio expressing physiological reactivity per unit plasma nicotine was negatively correlated with plasma cotinine level in smokers, reaching statistical significance for heart rate $(r=$ $-.69)$ and systolic blood pressure $(r=-.82)$, though not for diastolic pressure $(r=-.48)$. The finding of an inverse relationship between sensitivity to nicotine and intensity of prior exposure to nicotine was not unanticipated, as it was in keeping with various demonstrations of acute tolerance after nicotine dosing in minimally deprived smokers
(Hughes and Hatsukami, 1986; Perkins et al., 1993; Pomerleau et al., 1983).

While the finding that heavy smokers were less reactive to nicotine (i.e., they exhibited greater functional tolerance) than light smokers was expected, the observation that never-smokers as a whole were not much more reactive to nicotine than regular smokers was surprising-considering that the procedure was biased toward showing diminished nicotine reactivity in smokers due to tachyphylaxis (Porchet et al., 1988) caused by the lingering effects of nicotine from the cigarette smoked an hour earlier. To investigate further the possibility that tachyphylaxis might be masking underlying sensitivity in smokers - as had been suggested by West and Russell $(1985,1988)$-a comparison of the effects of a nicotine probe was undertaken in five male never-smokers (from the study described above) and an age-matched cohort of six light and six heavy smokers who had been 

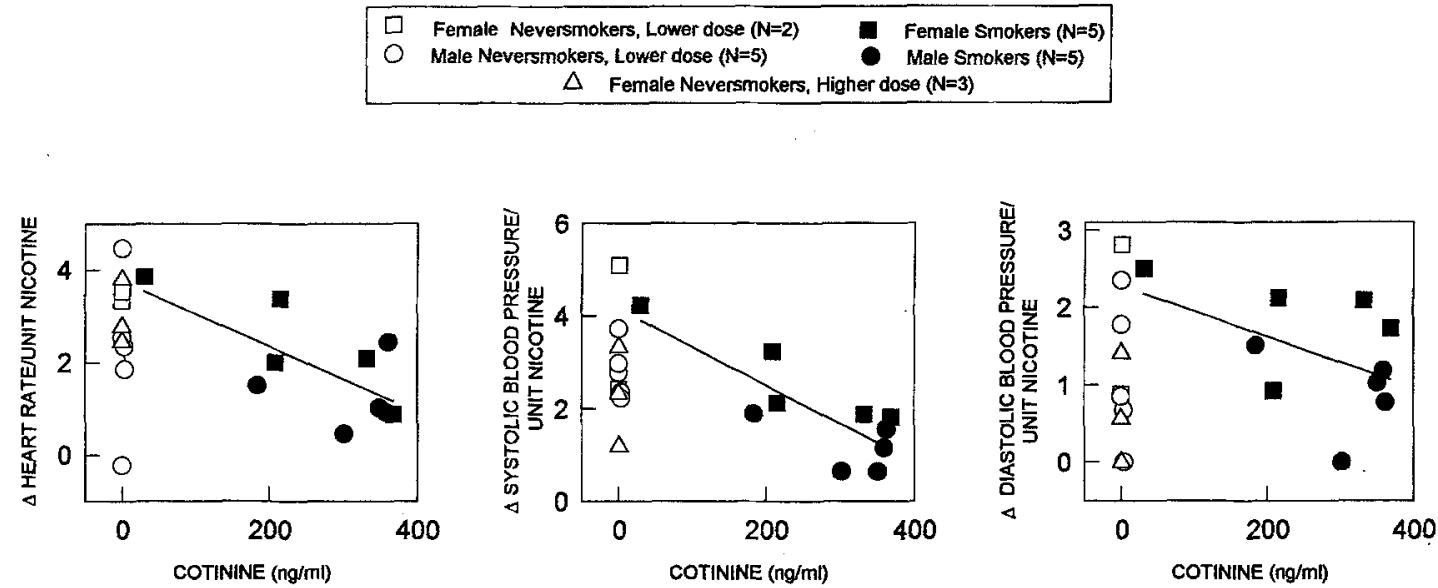

Fig. 4. Mean physiological reactivity (peak increment) per unit plasma nicotine (peak increment) for 10 smokers and 10 neversmokers as a function of plasma cotinine level (from Pomerleau et al., 1993b).

deprived overnight instead of being minimally deprived; since dosing was adjusted to take into account likely differences in pharmacokinetic tolerance between the smokers and the nonsmokers, the resulting plasma nicotine increments were nearly the same for the two groups. The findings corroborated the hypothesis that underlying differences in sensitivity to nicotine might be obscured by tachyphylaxis from recent smoking in undeprived smokers: After overnight deprivation (to allow sufficient time for the contribution of nicotine-agonist activity to dissipate), the heaviest smokers were also the most reactive to nicotine, the never-smokers were the least reactive, and light smokers fell between the other two groups (Pomerleau et al., 1992b).

These findings replicated and extended Russell's (1989) informal observation that, compared with less dependent smokers, smokers who are more dependent (i.e., those who experience more severe withdrawal symptomatology) are more sensitive to the first cigarette of the day after $24 \mathrm{~h}$ of abstinence. Further, an important feature of the present approach, is that, by utilizing the complete pharmacokinetic response to nicotine dosing (i.e., by determining the point of inflection for plasma nicotine concentration), it was possible to factor out the contribution of pharmacokinetic tolerance from that of pharmacodynamic tolerance in order to arrive at a more accurate estimate of functional sensitivity to nicotine for each subject.
The findings provide a plausible explanation for numerous other "anomalous" findings in literature. For example, Lombardo et al. (1988) had examined physiological reactivity in overnight-deprived smokers and found that more dependent smokers [classified using the Fagerström Tolerance Questionnaire; FTQ (Fagerström, 1978)] exhibited greater reactivity after smoking a usual cigarette than less dependent smokers; while the authors surmised that the results raised questions about the validity of the FTQ for measuring physical dependence, an alternative explanation is that dissipation of acute tolerance after overnight deprivation may have unmasked underlying differences in sensitivity to nicotine that set the stage for dependence in the first place. In a similar vein, Lee et al. (1987) examined the effects of a nicotine probe after various lengths of smoking deprivation and found that, after 7 days of abstinence, smokers were even more physiologically reactive to nicotine than after overnight deprivation.

Taken in conjunction with the animal research by Collins and his associates, the intranasal nicotine studies in humans suggest that preexisting differences in sensitivity to nicotine, rather than exposure to nicotine over time, determine subsequent nicotine use; that is, people who are "destined" to become heavy smokers may simply be more sensitive to nicotine's effects from the onset. A further inference is that, what passed as chronic functional tolerance in heavy smokers (based on 
earlier studies in which systematic measurements of plasma nicotine were not available to establish the peak) might simply be a physiological manifestation of acquired pharmacokinetic (metabolic) tolerance; in this case, diminished physiological reactivity would also be manifested, but for the reason that plasma levels for a given dose of nicotine were lowered by enhanced disposition or clearance of nicotine--an effect likely to be more pronounced in heavy smokers.

Recent observations in a category of smokers who show little evidence of nicotine dependence strengthen these suppositions. According to Shiffman and associates, about $5 \%$ of smokers clearly fail to meet the criteria for nicotine dependence (Shiffman, 1991b). Shiffman called these smokers "chippers," defined as long-term light smokers who smoke no more than five cigarettes per day at least 4 days per week. In contrast to regular smokers, one-fourth of chippers studied abstained from smoking as frequently as 1 day per month. When chippers did smoke, moreover, they often waited until later in the day, experiencing considerable deprivation without apparent discomfort or withdrawal; chippers inhaled and absorbed normal amounts of nicotine from each cigarette, however, revealing no signs of compensation for low frequency of smoking by taking in more nicotine from individual cigarettes (Shiffman et al., 1990). Overall, in contrast to regular smokers - and despite the fact that chippers had smoked nearly 50,000 cigarettes over an average of 20 years-chippers displayed a casual 'take-it-or-leave-it"' attitude toward smoking (Shiffman, 1989).

Of particular relevance to the present thesis, chippers were remarkably insensitive to nicotineexhibiting no greater reactivity to nicotine (no less tolerance) than a matched group of regular smokers, even though the regular smokers had consumed in excess of five times more cigarettes over their lifetime (Shiffman et al., 1992). Furthermore, there were no significant differences in nicotine half-life in plasma between chippers and regular smokers, indicating that the pattern of intermittent nicotine administration by chippers was not simply due to a greater accumulation of nicotine resulting from diminished nicotine disposition or clearance.

An analysis of smoking history, though retrospective and less precise than laboratory investigation, revealed that chippers' insensitivity to nicotine may have predated the first experience of smoking in that they reported fewer aversive experiences to the first exposure than regular smokers (Shiffman, 1989). Evidence suggesting that reaction to nicotine might have a genetic basis comes from studies of smoking in the parents and siblings of chippers (Gnys and Shiffman, 1991; Shiffman, 1989): Immediate relatives of chippers were just as likely to have smoked as relatives of regular smokers, but relatives of chippers were less nicotine dependent - they smoked less, were more likely to have been chippers themselves, and were more successful at quitting smoking, and some (e.g., sisters) reported fewer withdrawal symptoms on cessation. Finally, relatives of chippers indicated that the onset of smoking occurred later in the day compared with regular smokers (even controlling for amount smoked); this finding is especially revealing given that the latency between waking and the first cigarette of the day is considered the best single indicator of dependence (Fagerström, 1978; Kozlowski et al., 1981). Thus, in the aggregate, Shiffman's findings suggest that the ability to smoke without developing dependence may run in families.

\section{THE SENSITIVITY MODEL}

As indicated above, data from some recent animal and human experiments reveal difficulties with the exposure model as originally formulated. The findings suggest that individual differences in initial sensitivity to nicotine must play a much greater role in determining tolerance and dependence than has been taken into account. Accordingly, an alternative conceptualization, the sensitivity model, has been proposed (see review by Pomerleau et al., 1993a): Initial exposure to nicotine in individuals who have high innate sensitivity is seen as producing not only aversive effects but also highly reinforcing consequences, including temporary improvements in performance or affect along with termination of nicotine withdrawal (Pomerleau, 1981; Pomerleau and Pomerleau, 1984). As illustrated in Fig. 5, in such people, nicotine exposure quickly leads to the development of tolerance; smoking is rapidly entrained and is relatively independent of environmental support beyond availability of product and convenience, and discontinuation of smoking leads to loss of tolerance along with intense withdrawal symptoms. Once the habit is entrained, these people meet the 


\begin{tabular}{|c|c|c|}
\hline INITIAL SENSITIVITY & DEGREE OF TOLERANCE & SELF-ADMINISTRATION PATTERN \\
\hline $\begin{array}{l}\text { high sensitivity } \\
\text { (mix of aversive and } \\
\text { rewarding effects) }\end{array}$ & \begin{tabular}{|l|} 
exposure rapidly induces \\
tolerance to aversive effects
\end{tabular} & $\begin{array}{l}\text { high nicotine self-administration } \\
\text { (dependent smoker) }\end{array}$ \\
\hline
\end{tabular}

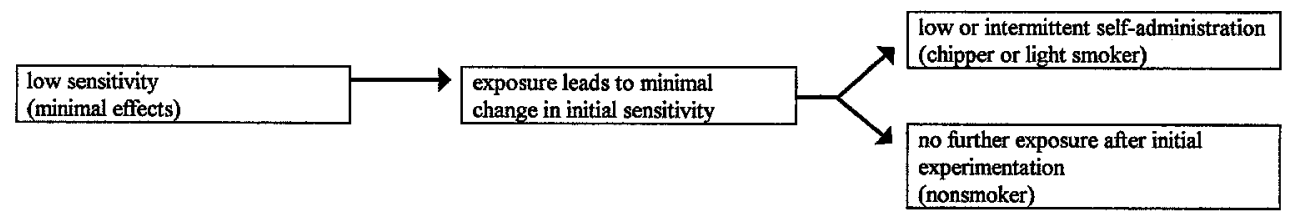

Fig. 5. Diagram of the sensitivity model (from Pomerleau et al., 1993a).

requirements for classification as highly dependent. On the other hand, people who are less sensitive to nicotine initially may experience less intense effects from nicotine, and their reactions to nicotine may be less susceptible to change in response to further exposure. Depending on prevailing environmental and social conditions, such people will remain nonsmokers or, in more supportive circumstances for smoking, will develop a pattern of minimal or intermittent nicotine administration that reflects the limited reinforcement they obtain from nicotine; these people meet the requirements for classification as minimally dependent.

The sensitivity model holds that constitutional ${ }^{3}$ factors, operative prior to nicotine exposure, determine smoking status by delimiting the extent of dependence possible for the individual. The development of acute functional tolerance to effects that are toxic or aversive - an adaptation that may be proportional to the magnitude of initial sensitivity-may constitute a dampening of sensitivity brought about by the disruption of homeostasis by nicotine. Smokers may be selected from a population of people who are highly sensitive to nicotine; furthermore, it is entirely possible that the enhancement of functional tolerance to the aversive effects of nicotine is not accompanied by an equivalent loss of sensitivity to its pleasurable or other reinforcing effects (see Robinson, 1993). Neversmokers, on the other hand, may be selected from a population of people who are relatively insensi-

\footnotetext{
${ }^{3}$ The word "constitutional" is used to take into account the possibility that prenatal exposure to nicotine (Slotkin et al., 1987; Zbuzek and Chin, 1994) might affect the subsequent development of dependence - thus, both genetic influences and intrauterine environmental exposure may contribute to sensitivity to nicotine.
}

tive to nicotine and, therefore, have limited capability to develop tolerance; these individuals fail to seek further exposure to nicotine, not because they are deterred by its aversive or toxic effects, but simply because they are not responsive to its blandishments. Chippers are the exception who prove the rule - relatively insensitive individuals who, by virtue of their lack of sensitivity, have no need to develop tolerance to dampen the perturbations caused by nicotine; as a result, they can take up smoking on an occasional basis and they do not become dependent. An important inference that can be taken from the human research summarized above is that, when individual differences are extensive - as they are among regular smokers, occasional smokers, and never-smokers-genetic variability is a likely explanation (Collins and Marks, 1989).

\section{Genetic Studies}

As described in a recent overview by McClearn et al. (1991), the study of genetic influences on behavior relied upon two main approaches in the past. The first was predicated on the search for single genes with influence sufficiently great as to be detectable against almost any genetic or environmental background; unfortunately, research quickly revealed that there were also large environmental influences in the expression of the behaviors involved in substance abuse. The second general approach was the application of statistical models of quantitative genetics to partition the variance of continuously distributed phenotypes into various fractions attributable to the actions of several genes-each with individually small effects - and 
the influence of environment. A third, more recent, approach involves the use of molecular genetics as a tool for studying genes with effects which, while not overwhelming, can, nonetheless, account for appreciable proportions of the variance. Substance abuse may be especially well suited for the last approach, given recent observations on genetic control in the expression of dopamine receptors and that activation of mesolimbic and mesocortical dopamine systems serves as a common pathway for a variety of addictive substances (Uhl et al., 1992).

In a provocative commentary on genetic research on substance abuse, Kozlowski and McClearn (1991) pointed out that, despite similar estimates of heritability for tobacco smoking and alcohol abuse, research on the human genetics of smoking has not developed as extensively. For instance, other than a brief mention that genes may contribute to individual differences in vulnerability to tobacco use, there is little discussion of genetic research in the Surgeon General's comprehensive review of nicotine addiction (USDHHS, 1988). According to Kozlowski and McClearn, a possible explanation is that Fisher's "constitutional hypothesis" (Fisher, 1959) may have discouraged genetic research on tobacco smoking due to "guilt by association": Fisher had argued that some gene or genes might be linked to both cancer and to smoking and concluded that smoking did not cause cancer but was "merely" a correlate. While the potential use of this argument to exonerate tobacco companies from responsibility for product liability was indeed unfortunate, overreaction to the hypothesis expressed in its extreme form may have retarded research on the genetics of smoking and nicotine dependence. Kozlowski and McClearn's (1991, p. 3) conclusion bears repetition: "It is not a case of 'nature versus nurture' in explaining variance in complex phenotypes; it is a case of nature and nurture, with the scientific question being the relative influence of these two general domains of influence on the trait under examination."

Global measures of heritability for complex behaviors such as smoking do not specify the mechanisms that may have contributed to observed concordance. A pair of twins, for example, may be concordant for smoking for a host of reasons. According to Kozlowski (1991), if genes contributed to making someone more sensitive to the acute toxic effects of smoking, this might cause not smoking to be inherited; if genes caused some in- dividuals to find nicotine's psychoactive effects reinforcing, however, this might also promote concordance for smoking. "These disparate encouraging and discouraging forces could then produce a high heritability of a global measure, without necessarily informing about the diverse, and possibly genetically-independent, functional systems contributing to the concordance"' (p. 519). Thus, the problem of what is inherited is of crucial importance: Knowledge about the genetic basis for nicotine's aversive and reinforcing effects is required for a satisfactory explanation of nicotine dependence. The remainder of the discussion seeks to identify a few approaches that seem promising.

\section{Implications of Neuroscience and Biobehavioral Research Findings for Genetic Studies}

It was said about Barbara McClintock, who won a Nobel Prize for her research on the genetics of the "jumping gene," that she had "a feeling for the organism." A similar "feel" for smoking by researchers on the genetics of nicotine and smoking is advisable. Because nicotine alters the bioavailability of numerous behaviorally and physiologically active neuroregulators, including acetylcholine, norepinephrine, dopamine, serotonin, $\beta$-endorphin, vasopressin, adrenocortiocotropic hormone (ACTH), and cortisol (Pomerleau and Rosecrans, 1989), nicotine can be "used" by smokers to produce a variety of effects, including temporary improvement in performance or affect. A potentially large number of exteroceptive and interoceptive cues unrelated to the nicotine-dependence cycle (i.e., smoking to terminate or to avoid withdrawal) can serve as discriminative stimuli for smoking; this may account for how the habit can become so thoroughly "interwoven into the fabric of daily living" (Pomerleau and Pomerleau, 1984). The rapid action of nicotine-along with its diverse neuroregulatory effects-makes it ideally suited to serve as a "pharmacological coping response"; it is available on demand and has effects that rarely outlast the circumstances that prompted its use. Oscar Wilde aptly conveyed the user's perspective some time ago: "Smoking is the perfect type of a perfect pleasure. It is exquisite, and leaves one unsatisfied. What more could one want?"

Dopamine (DA) pathways have attracted considerable attention in substance abuse research of 
late, in part because increased DA secretion seems to be a common factor in determining the addictive properties of most substances of abuse (Clarke, 1990b; Wise and Bozarth, 1987). Various drugs of abuse, including the "classic" psychomotor stimulants-cocaine and amphetamine-are potent stimulators of mesolimbic DA pathways, with concomitant enhancement of locomotor activity; though less potent, nicotine has similar actions. The reinforcing properties of these drugs-including nicotine-are also mediated by mesolimbic pathways (Balfour, 1993; Di Chiara and Imperato, 1988), and repeated daily administration causes sensitization (increased reactivity, or reverse tolerance) of behavioral activation (Clarke, 1990a; Kita et al., 1992; Robinson, 1993). An important consequence of sensitization of mesolimbic DA neurotransmission-described at some length in a recent theoretical paper by Robinson and Berridge (1993) - is that it enhances "incentive salience," hyperresponsivity to drug-associated stimuli as well as to the drug itself. The development of increased reactivity following exposure to a drug suggests a mechanism that might explain how people with high sensitivity to nicotine could develop dependence rapidly and extensively compared with people who are less sensitive (see Pomerleau et al., 1993a): Since the direction and rate of change in reactivity manifested as tolerance vary considerably across different response systems (Benowitz et al., 1982; Hasenfratz et al., 1990; Perkins et al., 1991; Russel1, 1989; USDHHS, 1988, pp. 47-49), there is the distinct possibility that the smoker destined to be highly dependent is the one who becomes sensitized (that is, develops bigger effects over time) to nicotine's reinforcing effects while becoming tolerant to nicotine's aversive effects. This hypothesis is testable and deserves consideration.

How a cigarette is smoked also can make a difference: Peak plasma nicotine concentration is typically reached around the time the cigarette is finished, and nicotine levels return to baseline after an hour or so. Russell (1990) has speculated that there may be two principal types of smokers: The first type, called the "peak seeker," is characterized as someone who seeks out rewarding or stimulating sensations-effects that are associated with activation of nicotine receptors via a rapid rise in plasma nicotine (Balfour, 1993). The second type, called the "trough maintainer," includes most heavy smokers, people who allow only short intervals between cigarettes. Compared with peak seekers, the smoking pattern of trough maintainers is not associated with marked peaks and troughs in nicotine concentration. Though trough maintainers may obtain less nicotine from each cigarette, plasma nicotine levels nonetheless continue to accumulate throughout the day, reaching an asymptote in late afternoon or early evening. For trough maintainers, the trigger to smoke may be a fall in plasma nicotine levels (reflecting diminishing nicotine concentrations in brain), constituting an interoceptive aversive stimulus that presages nicotine withdrawal and/or the loss of calming or sedative effects from the previous dose of nicotine; for peak seekers, however, the trigger may be an exteroceptive stimulus (e.g., an event that announces performance demand or dysphoric mood) that sets the occasion for pleasurable or favorable consequences from nicotine self-administration (see Pomerleau and Pomerleau, 1984). Chippers may be the ultimate example of peak-seekers, as they smoke intermittently and get positive effects without experiencing an aversive withdrawal cycle (see Shiffman et al., 1994). For most dependent smokers, though, one or the other pattern of smoking may predominate at various times, depending on environmental circumstances: intermittent, small doses may be taken in a context in which the stimulant or activating effects of nicotine are reinforcing; higher, more frequent dosing may occur in a context in which the calming or sedating effects of nicotine are reinforcing; these effects are superimposed on nicotine withdrawal cycle-characteristic for each individual-involving self-administration to avoid or escape an aversive abstinence state (Pomerleau and Pomerleau, 1984, pp. 508-509).

Behavioral and molecular genetics research, by incorporating relevant findings from neuroscience and biobehavioral research, has the potential for explaining - in a much more complete and satisfactory fashion than before- the manner in which drug, environment, and organism come together to create addiction. As mentioned above, twin studies have been conducted on the heritability of smoking in an attempt to define the gene-environment interface. The matched twin-pair design, for example, is a highly efficient way to assess the contribution of environment and heredity because it controls for genetic similarity while seeking to identify the sources (environmental, constitutional, etc.) for dis- 
cordance; a special feature is that relatively few discordant $M Z$ twin pairs are needed to identify environmental contributions. To date, however, twin studies have relied mostly on self-report rather than more objective determination of the phenotype-i.e., actual measurement of the subjective, behavioral, physiological, neuroendocrine, and biochemical response to nicotine (or to a nicotine analogue). Further, to characterize the nature of susceptibility to nicotine adequately, future explorations should examine not only dependent smokers who exhibit different patterns of smoking (e.g., peak seekers versus trough maintainers), but nondependent smokers, exsmokers, and never-smokers. By extension, investigations of twin pairs exhibiting known cofactors for smoking such as depression (Kendler et al., 1993b) and other psychiatric states (Glassman, 1993) should be useful in identifying response tendencies that serve to increase the probability or the intensity of nicotine dependence. Cofactors may be a limiting condition for quitting smoking in an increasing proportion of people in countries where active public health campaigns against smoking are operative - the people who are the first to give up smoking are, by definition, the "easy quitters" (Glassman, 1993).

Research on the neural pathways involved in drug reinforcement, such as the dopamine reward system mentioned above, shows great potential for generating important clues about genotypic markers that might underlie the behavioral expression of substance abuse (Uhl et al., 1992). In particular, critical differences in the structure or expression of relevant genes may account for a substantial proportion of the variance in dependence or in degree of addiction. Moreover, the possibility of using dopamine agonists or antagonists as probes for studying reinforcement-relevant responses such as behavioral activation in people who are at the extremes of nicotine dependence should be considered. Either of these approaches - genotyping or drug probes-might lead to more accurate specification of potential for tolerance and dependence at the individual level. Furthermore, these approaches could provide the basis for new technologies for assessing likelihood of initiation of smoking or even ability to quit smoking. The effectiveness of prevention programs might be improved significantly, since biobehavioral susceptibility to nicotine dependence could be predicted and characterized. Among the possibilities for intervention and management are modification of response tendencies that enhance susceptibility in individuals who are vulnerable (e.g., remediation of traits or cofactors by behavioral or pharmacologic interventionor even gene therapy to modify sensitivity to nicotine's reinforcing effects) as well as the creation of alternatives to or substitutes for some of nicotine's neurochemical effects.

\section{CONCLUSION}

It may be that the abuse liability of a given drug is determined by the extent to which sensitivity to that drug resides in the gene pool (Pomerleau et al., 1993a). The current paucity of techniques to counter the contribution of organismic variables in substance abusers may explain, in part, why prevention strategies that rely principally on attempts to change social support for drug taking (e.g., public education and "just say no" campaigns) have failed to eradicate drug abuse. If the tenets of the sensitivity model are correct, modification of social support and other environmental contingencies has an impact largely on individuals who are relatively drug insensitive and, therefore, principally at risk of becoming light or intermittent users; people who are highly reactive and presumably highly vulnerable to drug use, however, will require a different approach. Moreover, when societal strictures for drug use become more severe, a shift in the balance of the nature/nurture equation is effected, because people for whom drug-taking is less reinforcing are selected out first; for those people who remain users after such interventions, therefore, environmental sanctions will have to be supplemented by special procedures to counter genetic predisposition.

\section{ACKNOWLEDGMENTS}

The author acknowledges with gratitude helpful comments by Cynthia S. Pomerleau, Ph.D., and Gary Swan, Ph.D.

\section{REFERENCES}

Alexander, B. K., and Hadaway, P. F. (1982). Opiate addiction: The case for an adaptive orientation. Psychol. Bull. 92:367-381.

American Psychiatric Association (1994). Diagnostic and Statistical Manual of Mental Disorders, 4th ed. (DSM-IV), American Psychiatric Association, Washington, DC. 
Balfour, D. J. K. (1993). The pharmacology of nicotine in the CNS and its bearing on nicotine replacement therapies. Int. J. Smoking Cessat. 2:3-9.

Benowitz, N. L. (1991). Importance of nicotine metabolism in understanding the human biology of nicotine. In Adlkofer, F, and Thurau, $\mathbf{K}$. (eds.), Effects of Nicotine on Biological Systems, Birkhäuser Verlag, Berlin, pp. 19-24.

Benowitz, N. L., Jacob, P., Jones, R. T., and Rosenberg, J. (1982). Interindividual variability in the metabolism and cardiovascular effects of nicotine in man. J. Pharmacol. Exp. Ther. 221:368-372.

Benwell, M. E. M., Balfour, D. J. K., and Anderson, J. M. (1988). Evidence that tobacco smoking increases the density of $(-)-\left[{ }^{3} \mathrm{H}\right]$ nicotine binding sites in human brain. $J$. Neurochem. 50:1243-1247.

Bewley, B. R., Bland, J. M., and Harris, R. (1974). Factors associated with the starting of cigarette smoking by primary school children. Br. J. Prev. Soc. Med. 28:37-44.

Carmelli, D., Swan, G. E., Robinette, D., and Fabsitz, R. R. (1990). Heritability of substance use in the NAS-NRTC twin registry. Acta Genet. Med. Gemellol. 39:91-98.

Carmelli, D., Swan, G. E., Robinette, D., and Fabsitz, R. (1992). Genetic influence on smoking-A study of male twins. N. Engl. J. Med. 327:829-833.

Clarke, P. B. S. (1990a). Dopaminergic mechanism in the locomotor stimulant effects of nicotine. Biochem. Pharmacol. 40:1427-1432.

Clarke, P. B. S. (1990b). Mesolimbic dopamine activationthe key to nicotine reinforcement. In Bock, G., and Marsh, J. (eds.), The Biology of Nicotine Dependence (Ciba Foundation Symposium 152), John Wiley, Chichester, pp. 153-168.

Collins, A. C., (1990). Interactions of ethanol and nicotine at the receptor level. In Galanter, M. (ed.), Combined Alcohol and Other Drug Dependence, Vol. VIII, Plenum Press, New York.

Collins, A. C., and Marks, M. J. (1989). Chronic nicotine exposure and brain nicotinic receptors-influence of genetic factors. Prog. Brain Res. 79:137-146.

Collins, A. C., and Marks, M. J. (1991). Progress towards the development of animal models of smoking-related behaviors. J. Addict. Dis. 10:109-126.

Comings, D. E., Ferry, L., Bradshaw-Robinson, S., Burchette, R., Dino, M., Chiu, C., and Muhleman, D. (1993). Role of variants of the dopamine $\mathrm{D}_{2}$ receptor (DRD2) gene as genetic risk factors in smoking. Paper presented at the First Annual Scientific Conference, Tobacco-Related Research Program, University of California, San Francisco.

Di Chiara, G., and Imperato, A. (1988). Drugs abused by humans preferentially increase synaptic dopamine concentrations in the mesolimbic dopamine system of freely moving rats. Proc. Natl. Acad. Sci. USA 85:5274-5278.

Eaves. L. J., and Eysenck, H. J. (1980). New approaches to the analysis of twin data and their application of smoking behavior. In Eysenck, H. J. (ed.), The Causes and Effects of Smoking, Maurice Temple Smith, London, pp. 140-314.

Fagerström, K. O. (1978). Measuring degree of physical dependence to tobacco with reference to individualization of treatment. Addict. Behav. 3:235-241.

Fisher, R. A. (1958a). Cancer and smoking. Nature 182:596.

Fisher, R. A. (1958b). Lung cancer and cigarettes. Nature 182: 108.

Fisher, R. A. (1959). Smoking: The Cancer Controversy. Some Attempts to Assess the Evidence, Oliver and Boyd, London.

Friedman, L. S., Lichtenstein, E., and Biglan, A. (1985). Smoking onset among teens: An empirical analysis of initial situations. Addict. Behav. 10:1-13.
Glassman, A. H. (1993). Cigarette smoking: Implications for psychiatric illness. Am. J. Psychiatry 150:546-553.

Gnys, M., and Shiffman, S. (1991). Familial contributions to nicotine dependence. In Shiffman, S. (Chair), Chippers: Studies of Non-Dependent Cigarette Smokers, Symposium presented at the annual meeting of the Society of Behavioral Medicine, Washington, DC.

Goldman, D., Deneris, E., Luyten, W., Kochhar, A., Patcick, J., and Heinemann, S. (1987). Members of a nicotinic acetylcholine receptor gene family are expressed in different regions of mammalian central nervous system. Cell 48:965-973.

Greden, J. F., and Pomerleau, O. F. (in press). Caffeine and nicotine. In Kaplan, N. I., and Sadock, B. J. (eds.), Comprehensive Textbook of Psychiatry, 6th ed., Williams and Wilkins, Baltimore.

Gurling, H. M. D., Grant, S., and Dangl, J. (1985). The genetic and cultural transmission of alcohol use, alcoholism, cigarette smoking and coffee drinking: A review and an example using a log linear cultural transmission model. $B r$. J. Addict. 80:269-279.

Hannah, M. C., Hopper, J. L., and Mathews, J. D. (1984). Twin concordance for a binary trait. II. Nested analysis of eversmoking and ex-smoking traits and unnested analysis of a committed-smoking trait. Am. J. Hum. Genet. 37:153165.

Hasenfratz, M., Nil, R., and Battig, K. (1990). Development of central and peripheral smoking effects over time. $P s y-$ chopharmacology 101:359-365.

Heath, A. C., and Martin, N. G. (1993). Genetic models for the natural history of smoking: Evidence for a genetic influence on smoking persistence. Addict. Behav, 18:19-34.

Hirschman, R. S., Leventhal, H., and Glynn, K. (1984). The development of smoking behavior: Conceptualization and supportive cross-sectional survey data. J. Appl. Soc. Psychol. 14:184-206.

Hughes, J. R. (1986). Genetics of smoking: A brief review. Behav. Ther. 17:335-345.

Hughes, J. R., and Hatsukami, D. K. (1986). Signs and symptoms of tobacco withdrawal. Arch. Gen. Psychiatry 43: 289-294.

Hughes, J. R., Gust, S. W., and Pechacek, T. F. (1987). Prevalence of tobacco dependence and withdrawal. Am. J. Psychiatry 144:205-208.

Kendler, K. S., Heath, A. C., Neale, M. C., Kessler, R. C., and Eaves. L. J. (1993a). Alcoholism and major depression in women: A twin study of the causes of comorbidity. Arch. Gen. Psychiatry 50:690-698.

Kendler, K. S., Neale, M. C., MacLean, C. J., Heath, A. C., Eaves, L. J., and Kessler, R. C. (1993b). Smoking and major depression: A causal analysis. Arch. Gen. Psychiatry 50:36-43.

Killen, J. D., Fortmann, S. P., Telch, M. J., and Newman, B. (1988). Are heavy smokers different from light smokers? JAMA 260:1581-1585.

Kita, T., Okamoto, M., and Nakashima, T. (1992). Nicotineinduced sensitization to ambulatory stimulant effect produced by daily administration into the ventral tegmental area and nucleus accumbens in rats. Life Sci. 50:583-590.

Kozlowski, L. T. (1980). The role of nicotine in the maintained use of cigarettes. Drug Merchandiz. (Canada) January: $36-43$.

Kozlowski, L. T. (1991). Rehabilitating a genetic perspective in the study of tobacco and alcohol use. Br. J. Addict. 86: $517-520$.

Kozlowski, L. T., and McClearn, G. E. (1991). "Cross-cultural comparison" of the genetic perspective in the study of tobacco and alcohol use. Poster presentation at the annual 
meeting of the Society of Behavioral Medicine, Washington, DC.

Kozlowski, L. T., Director, J., and Harford, M. A. (1981). Tobacco dependence, restraint and time to the first cigarette of the day. Addict. Behav. 6:307-312.

Lee, B. L., Benowitz, N. L., and Jacob, P. (1987). Influence of tobacco abstinence on the disposition kinetics and effects of nicotine. Clin. Pharmacol. Ther. 41:474-479.

Lombardo, T. W., Hughes, J. R., and Fross, J. D. (1988). Failure to support the validity of the Fagerström Tolerance Questionnaire as a measure of physiological tolerance to nicotine. Addict. Behav. 13:87-90.

Marks, M. J., and Collins, A. C. (1982). Characterization of nicotine binding in mouse brain and comparison with the binding of alpha-bungarotoxin and quinuclidinyl benzilate. Mol. Pharmacol. 22:554-564.

Marks, M. J., Stitzel, J. A., and Collins, A. C. (1986). A doseresponse analysis of nicotine tolerance and receptor changes in two inbred mouse strains. J. Pharmacol. Exp. Ther. 239:358-364.

Marks, M. J., Romm, E., Campbell, S. M., and Collins, A. C. (1989a). Variation of nicotinic binding sites among inbred strains. Pharmacol. Biochem. Behav. 33:679-689.

Marks, M. J., Stitzel, J. A., and Collins, A. C. (1989b). Genetic influences on nicotine responses. Pharmacol. Biochem. Behav. 33:667-668.

McClearn, G. E. Plomin, R., Gora-Maslak, G., and Crabbe, J. C. (1991). The gene chase in behavioral science. Psychol. Sci. 2:222-229.

McNeil, A. D. (1991). The development of dependence on smoking in children. Br. J. Addict. 86:589-592.

Murphree, H. B. (1979). EEG effects in humans of nicotine, tobacco smoking, withdrawal from smoking and possible surrogates. In Redmond, A., and Izard, C. (eds.), Electrophysical Effects of Nicotine, Elsevier/North-Holland Biomedical Press, Amsterdam, pp. 227-243.

Noble, E. P. (1993). The $\mathrm{D}_{2}$ dopamine receptor gene: $\mathrm{A}$ review of association studies in alcoholism. Behav. Genet. 23: 119-129.

Ochoa, E. L. M., Li, L., and McNamee, M. G. (1990). Desensitization of central cholinergic mechanisms and neuroadaptation to nicotine. Mol. Neurobiol. 4:251-287.

Perkins, K. A., Stiller, R. L., and Jennings, J. R. (1991). Acute tolerance to the cardiovascular effects of nicotine. Drug Alcohol Depend. 29:77-85.

Perkins, K. A., Grobe, J. E., Epstein, L. H., Caggiula, A., Stiller, R. L., and Jacob, R. G. (1993). Chronic and acute tolerance to subjective effects of nicotine. Pharmacol. Biochem. Behav. 45:375-381.

Pomerleau, O. F. (1981). Underlying mechanisms in substance abuse: Examples from research on smoking. Addict. Behav. 6:187-196.

Pomerleau, O. F., and Pomerleau, C. S. (1984). Neuroregulators and the reinforcement of smoking: Towards a biobehavioral explanation. Neurosci. Biobehav. Rev. 8:503513.

Pomerleau, O. F., and Rosecrans, J. (1989). Neuroregulatory effects of nicotine. Psychoneuroendocrinology 14:407423.

Pomerleau, O. F., Fertig, J. B., and Shanahan, S. O. (1983). Nicotine dependence in cigarette smoking: An empirically-based, multivariate model. Pharmacol. Biochem. Behav. 19:291-299.

Pomerleau, O. F., Pomerleau, C. S., and Rose, J. E. (1989). Controlled dosing of nicotine: Problems and progress. Ann. Behav. Med. 11:158-163.

Pomerleau, O. F., Flessland, K. A., Pomerleau, C. S., and Hariharan, M. (1992a). Controlled dosing of nicotine via an intra-nasal nicotine aerosol delivery device (INADD). Psychopharmacology 108:519-526.

Pomerleau, O. F., Pomerleau, C. S., Cameron, O. G., and Hariharan, M. (1992b). Sensitivity to nicotine in smokers and never-smokers. Proceedings of the 54th Annual Scientific Meeting, Committee on Problems on Drug Dependence, Inc. (NIDA Research Monograph No. 132, USDHHS), National Institute on Drug Abuse, Rockville MD.

Pomerleau, O. F., Collins, A. C., Shiffman, S., and Pomerleau, C. S. (1993a). Why some people smoke and others do not: New perspectives. J. Consult. Clin. Psychol. 61:723731

Pomerleau, O. F., Hariharan, M., Pomerleau, C. S., Cameron, O. G., and Guthrie, S. K. (1993b). Differences between smokers and never-smokers in sensitivity to nicotine: A preliminary report. Addiction 88:113-118.

Porchet, H. C., Benowitz, N. L., Sheiner, L. B., and Copeland, J. R. (1987). Apparent tolerance to the acute effect of nicotine results in part from distribution kinetics. $J$. Clin. Invest. 80:1466-1471.

Porchet, H. C., Benowitz, N. L., and Sheiner, L. B. (1988). Pharmacodynamic model of tolerance: Application to nicotine. J. Pharmacol. Exp. Ther. 244:231-236.

Presti, D. E., Ary, D. V., and Lichtenstein, E. (1992). The context of smoking initiation and maintenance: Findings from interviews with youths. J. Subst. Abuse 4:35-45.

Robinson, T. E. (1993). Persistent sensitizing effects of drugs on brain dopamine systems and behavior: Implications for addiction and relapse. In Korenman, S., and Barchas, J. P. (eds.), The Biological Basis of Substance Abuse, Oxford University Press, New York, pp. 373-402.

Robinson, T. E., and Berridge, K. C. (1993). The neural basis of drug craving: An incentive-sensitization theory of addiction. Brain Res. Rev. 18:247-291.

Russell, M. A. H. (1978). Self-regulation of nicotine intake by smokers. In Battig, K. (ed.), Behavioral Effects of Nicotine, S. Karger, Basel, pp. 108-122.

Russell, M. A. H. (1989). Subjective and behavioural effects of nicotine in humans: Some sources of individual variation. In Nordberg, A., Fuxe, K., Holmstedt, B., and Sundwall, A. (eds.), Progress in Brain Research, Vol. 79, Elsevier Science, New York, pp. 289-302.

Russell, M. A. H. (1990). Nicotine intake and its control over smoking. In Wonnacott, S., Russell, M. A. H., and Stolerman, I. P. (eds.), Nicotine Psychopharmacology: Molecular, Cellular and Behavioural Aspects, Oxford Scientific Publications, Oxford, UK, pp. 374-418.

Shiffman, S. (1989). Tobacco "chippers": Individual differences in tobacco dependence. Psychopharmacology 97: 539-547.

Shiffman, S. (1991a). Chippers: Characteristics of non-dependent smokers. In Shiffman, S. (Chair), Chippers: Studies of Non-dependent Cigarette Smokers, Symposium presented at the annual meeting of the Society of Behavioral Medicine, Washington, DC.

Shiffman, S. (1991b). Refining models of dependence: Variations across persons and situations. Br. J. Addict. 86:611615.

Shiffman, S., Fischer, L. B., Zettler-Segal, M., and Benowitz, N. L. (1990). Nicotine exposure among nondependent smokers. Arch. Gen. Psychiatry 47:333-336.

Shiffman, S., Zettler-Segal, M., Kassel, J., Paty, J., Benowitz, N. L., and O'Brien, G. (1992). Nicotine elimination and tolerance in non-dependent cigarette smokers. Psychopharmacology 109:449-456.

Shiffman, S., Kassel, J. D., Paty, J., Gnys, M., and ZettlerSegal, M. (1994). Smoking typology profiles of chippers and regular smokers. J. Subst. Abuse 6:21-35. 
Silverstein, B., Kelly, E., Swan, J., and Kozlowski, L. T. (1982). Physiological predisposition toward becoming a cigarette smoker: Experimental evidence for a sex difference. Addict. Behav. 7:83-86.

Slotkin, T. A., Cho, H., and Whitmore, W. L. (1987). Effects of prenatal nicotine exposure on neuronal development: Selective actions on central and peripheral catecholaminergic pathways. Brain Res. Bull. 18:601-611.

Smith, S. S., O'Hara, B. F., Persico, A. M., Gorelick, D. A., Newlin, D. B., Vlahov, D., Solomon, L., Pickens, R., and Uhl, G. R. (1992). Genetic vulnerability to drug abuse: The $\mathrm{D}_{2}$ receptor Taq $\mathrm{B} 1$ restriction fragment length polymorphism appears more frequently in polysubstance abusers. Arch. Gen. Psychiatry 49:723-727.

Srivastava, E. D., Russell, M. A. H., Feyerabend, C., Masterson, J. G., and Rhodes, J. (1991). Sensitivity and tolerance to nicotine in smokers and nonsmokers. Psychopharmacology 105:63-68.

Swan, G. E., Carmelli, D., Rosenman, R. H., Fabsitz, R., and Christian, J. C. (1990). Smoking and alcohol consumption in adult male twins: Genetic heritability and shared environmental influences. J. Subst. Abuse 2:39-50.

Swan, G. E., Cardon, L. R., and Carmelli, D. (1994). The consumption of tobacco, alcohol, and caffeine in male twins:
A multivariate genetic analysis. Ann. Behav. Med. 16(Supplement):5069.

Uhl, G. R., Persico, A. M., and Smith, S. S. (1992). Current excitement with $\mathrm{D}_{2}$ dopamine receptor gene alleles in substance abuse. Arch. Gen. Psychiatry 49:157-160.

United States Department of Health and Human Services (1988). The Health Consequences of Smoking: Nicotine Addiction (1988 Report of the Surgeon General, Public Health Service, Office on Smoking and Health, Rockville, $\mathrm{MD}$.

West, R. J., and Russell, M. A. H. (1985). Pre-abstinence smoke intake and smoking motivation as predictors of severity of cigarette withdrawal symptoms. Psychopharmacology 87:334-336.

West, R. J., and Russell, M. A. H. (1988). Loss of acute nicotine tolerance and severity of cigarette withdrawal. Psychopharmacology 94:563-565.

Wise, R. A., and Bozarth, M. A. (1987). A psychomotor stimulant theory of addiction. Psychol. Rev. 94:469-492.

Zbuzek, V. K., and Chin, C. W. Y. (1994). Prenatal nicotine exposure increased duration of nicotine-induced analgesia in adult rats. Psychopharmacology 113:534 538. 